\title{
Portfolio Optimization without the Self-Financing Assumption
}

\author{
Moawia Alghalith \\ Department of Economics, University of the West Indies, St. Augustine, Trinidad and Tobago \\ E-mail:malghalith@gmail.com \\ Received January 13, 2011; revised February 28, 2011; accepted March 5, 2011
}

\begin{abstract}
In this paper, we relax the assumption of a self-financing strategy in the dynamic investment models. In so doing we provide smooth solutions and constrained viscosity solutions.
\end{abstract}

Keywords: Portfolio, Investment, Stochastic, Viscosity Solutions, Self Financing

\section{Introduction}

The literature on dynamic portfolio optimization is vast. However, previous literature on dynamic investment relied on the assumption of a self-financing strategy; that is, the investor cannot add or withdraw funds during the trading horizon. Examples include [1], [2], [3] and [4] among many others. However, this assumption is somewhat restrictive and sometimes unrealistic.

Moreover, even with the assumption of a self-financing strategy, the previous literature usually provided explicit solutions under the assumption of a logarithmic or power utility function. Therefore, the assumption of a self- financing strategy did not offer a significant simplification of the solutions. Therefore, the self-financing assumption needs to be relaxed.

Consequently, the goal of this paper is to relax the assumption of self-financing strategies. In this paper, we show that the assumption of a self-financing strategy can be relaxed without a significant complication of the optimal solutions. In so doing, we present a stochastic-fac- tor incomplete-markets investment model and provide both smooth solutions and constrained viscosity solutions.

\section{The Model}

We consider an investment model, which includes a risky asset, a risk-free asset and a random external economic factor (see, for example, [5]). We use a three-dimensional standard Brownian motion $\left\{W_{1 \mathrm{~s}}, W_{2 \mathrm{~s}}\right.$, $\left.W_{3 \mathrm{~s}}, F_{s}\right\}_{t \leq s \leq T}$ on the probability $\operatorname{space}\left(\Omega, F_{s}, P\right)$, where $\left\{F_{s}\right\}_{0 \leq s \leq T}$ is the augmentation of filtration. The risk-free asset price process is $S_{0}=e^{\int^{T} r\left(Y_{s}\right) \mathrm{d} s}$, where $r\left(Y_{s}\right)$ ä $C_{b}^{2}(R)$ is the rate of return and $Y_{s}$ is the stochastic economic factor.

The dynamics of the risky asset price are given by

$$
\mathrm{d} S_{s}=S_{s}\left\{\mu\left(Y_{s}\right) \mathrm{d} s+\sigma_{1}\left(Y_{s}\right) \mathrm{d} W_{1 s}\right\},
$$

where $\mu\left(Y_{s}\right)$ and $\sigma_{1}\left(Y_{s}\right)$ are the rate of return and the volatility, respectively. The economic factor process is given by

$$
\mathrm{d} Y_{s}=b\left(Y_{s}\right) \mathrm{d} s+\sigma_{2}\left(Y_{s}\right) \mathrm{d} W_{2 s}, Y_{t}=y,
$$

where $\sigma_{2}\left(Y_{s}\right)$ is its volatility and $b\left(Y_{s}\right)$ ä $C^{1}(R)$.

The amount of money added to or withdrawn from the investment at time $s$ is denoted by $\Phi_{s}$, and its dynamics are given by

$$
\mathrm{d} \Phi_{s}=a\left(Y_{s}\right) \mathrm{d} s+\sigma_{3}\left(Y_{s}\right) \mathrm{d} W_{3 s},
$$

where $\sigma_{3}\left(Y_{s}\right)$ is its volatility and $a\left(Y_{s}\right) b\left(Y_{s}\right)$ ä $C^{1}(R)$. Thus the wealth process is given by

$$
\begin{aligned}
X_{T}^{\pi}= & x+\int_{t}^{T} a\left(Y_{s}\right) \mathrm{d} s+\int_{t}^{T} \sigma_{3}\left(Y_{s}\right) \mathrm{d} W_{3 s} \\
& +\int_{t}^{T}\left\{r\left(Y_{s}\right) X_{s}^{\pi}+\left(\mu\left(Y_{s}\right)-r\left(Y_{s}\right) \pi_{s}\right)\right\} \mathrm{d} s \\
& +\int_{t}^{T} \pi_{s} \sigma_{1}\left(Y_{s}\right) \mathrm{d} W_{1 s},
\end{aligned}
$$

where $x$ is the initial wealth, $\left\{\pi_{s}, F_{s}\right\}_{t \leq s \leq T}$ is the portfolio process with $E \int_{t}^{T} \sigma_{1}^{2}\left(Y_{s}\right) \pi_{s}^{2} \mathrm{~d} s<\infty$.

The investor's objective is to maximize the expected 
utility of the terminal wealth

$$
V(t, x, y)=\sup _{\pi_{t}} E\left[u\left(X_{T}^{\pi}\right) \mid F_{t}\right],
$$

where $V($.$) is the value function, u($.$) is a$ differentiable, bounded and concave utility function.

Under regularity conditions, the value function is differentiable and thus satisfies the Hamiltonian-JacobiBellman PDE

$$
\begin{aligned}
& V_{t}+[r(y) x+a(y)] V_{x}+b(y) V_{y} \\
& +\frac{1}{2} \sigma_{2}^{2}(y) V_{y y}+\rho_{23} \sigma_{2}(y) \sigma_{3}(y) V_{x y}+\frac{1}{2} \sigma_{3}^{2}(y) V_{x x} \\
& +\sup _{\pi_{t}}\left\{\pi_{t}(\mu(y)-r(y)) V_{x}\right. \\
& \quad+\left[\frac{1}{2} \pi_{t}^{2} \sigma_{1}^{2}(y)+\rho_{13} \sigma_{1}(y) \sigma_{3}(y) \pi_{t}\right] V_{x x} \\
& \left.\quad+\rho_{12} \sigma_{1}(y) \sigma_{2}(y) \pi_{t} V_{x y}\right\} \\
& =0,
\end{aligned}
$$

$$
V(T, x, y)=u(x)
$$

where $\rho_{i j}$ is the correlation coefficient between the Brownian motions. Hence, the optimal solution is

$$
\begin{aligned}
\pi_{t}^{*}= & -\frac{(\mu(y)-r(y)) V_{x}+\rho_{12} \sigma_{1}(y) \sigma_{2}(y) V_{x y}}{\sigma_{1}^{2}(y) V_{x x}} \\
& -\rho_{13} \sigma_{1}^{-1}(y) \sigma_{3}(y) .
\end{aligned}
$$

Similar to the previous literature, an explicit solution can be obtained for specific forms of utility such as a logarithmic utility function.

\section{Viscosity Solutions}

We can apply the constrained viscosity solutions to (6), given the HJB is degenerate elliptic and monotone increasing in $V$ (see, for example, [6]).

Consider this HJB

$$
\begin{aligned}
H\left(x, V(x), V_{x}(x), V_{x x}(x)\right) & =0, x \text { ä } \Omega, \\
V(x) & =g(x), x \text { ä } \partial \Omega,
\end{aligned}
$$

where $\Omega$ is a bounded open set.

Definition 1 A continuous function $V(x)$ is a viscosity subsolution of (6) if

$$
\begin{aligned}
& H(x, V(x), P, X) \leq 0, \forall P \text { ä } D^{+} V(x), \\
& \forall X \text { 滗 } J^{+} V(x), \forall x \quad \Omega
\end{aligned}
$$

A continuous function $V(x)$ is a viscosity supersolution of (8) if

$$
\begin{aligned}
& H(x, V(x), P, X) \geq 0, \forall P \text { ä } D^{-} V(x), \\
& \forall X \text { 滗 } J^{-} V(x), \forall x \quad \Omega,
\end{aligned}
$$

where

$$
\begin{aligned}
& D^{+} V(x)=\left\{P: \lim _{y \rightarrow x} \sup \frac{V(y)-V(x)-\langle P, y-x\rangle}{|y-x|} \leq 0\right\}, \\
& D^{-} V(x)=\left\{P: \liminf _{y \rightarrow x} \frac{V(y)-V(x)-\langle P, y-x\rangle}{|y-x|} \geq 0\right\},
\end{aligned}
$$

are the super-differential and sub-differential, respectively; and

$$
\begin{aligned}
& J^{2+} V(x)=\{(P, X): \\
& \limsup _{y \rightarrow x} \frac{V(y)-V(x)-\langle P, y-x\rangle-\frac{1}{2}\langle X(y-x), y-x\rangle}{|y-x|} \\
&\leq 0\}, \\
& J^{2-} V(x)=\{(P, X): \\
& \liminf _{y \rightarrow x} \frac{V(y)-V(x)-\langle P, y-x\rangle-\frac{1}{2}\langle X(y-x), y-x\rangle}{|y-x|} \\
&\geq 0\},
\end{aligned}
$$

are the superject and subject, respectively. A function $V(x)$ is a viscosity solution if it is both a viscosity subsolution and a viscosity supersolution.

Proposition $1 \quad V(x)$ is the unique constrained viscosity solution of (6).

Proof Let $V$ ä $C(\partial \Omega)$ and let $s(V)$ and $i(V)$ be the upper and lower semicontinuous envelopes of $V$, respectively, where

$$
\begin{aligned}
& s(V)=\sup \left\{u(x): u_{1} \leq u \leq u_{2}\right\}, \\
& i(V)=\inf \left\{u(x): u_{1} \leq u \leq u_{2}\right\},
\end{aligned}
$$

where $u_{1}$ and $u_{2}$ are sub-solution and super-solution, respectively.

Thus $s(V)$ ä $U S C(\bar{\Omega})$ and $i(V)$ ä $\operatorname{LSC}(\bar{\Omega})$ are a viscosity subsolution and supersolution, respectively. At the boundary we have

$$
V(x)=s(V)=i(V),
$$

by the comparison principle

$$
s(V) \leq i(V) \text { in } \Omega .
$$

By definition $s(V) \geq i(V)$ and 
thus

$$
V(x)=s(V)=i(V) \text { in } \bar{\Omega}
$$

is the unique viscosity solution.

\section{References}

[1] M. Alghalith, "A New Stochastic Factor Model: General Explicit Solutions," Applied Mathematics Letters, Vol. 22, No. 12, 2009, pp. 1852-1854. doi:10.1016/j.aml.2009.07.011

[2] N. Castaneda-Leyva and D. Hernandez-Hernandez, "Optimal Consumption-Investment Problems in Incomplete Markets with Random Coefficients," Proceedings of the 44th IEEE Conference on Decision and Control, and the
European Control Conference 2005, Sevilla, 12-15 December 2005, pp. 6650-6655.

[3] J. Cvitanic and F. Zapatero, "Introduction to the Economics and Mathematics of Financial Markets," MIT Press, Cambridge, 2004.

[4] W. Fleming, "Some Optimal Investment, Production and Consumption Models," Contemporary Mathematics, Vol. 351, 2004, pp. 115-123.

[5] F. Focardi and F. Fabozzi, "The Mathematics of Financial Modeling and Investment Management," Wiley E-Series, 2004.

[6] F. Minani, "Hausdorff Continuous Viscosity Solutions to Hamilton-Jacobi Equations and their Numerical Analysis," Unpublished Ph.D. Thesis, University of Pretoria, Pretoria, 2007. 